\title{
Using foresight to improve the efficiency of process equipment design
}

\author{
Sergey Nikitin ${ }^{1, *}$, Arthur Novikov ${ }^{2}$ and Tatyana Novikova ${ }^{2}$ \\ ${ }^{1}$ Voronezh State Technical University, 14, Moscow Avenue, 394026, Voronezh, Russia \\ ${ }^{2}$ Voronezh State University of Forestry and Technologies named after G.F. Morozov, 8, Timiryazeva \\ Street, 394087, Voronezh, Russia
}

\begin{abstract}
The study is intended for engineers designing hybrid systems. The analytic hierarchy method was used as the main tool of foresight. Nowadays, some experience in the design of optoelectronic systems is accumulated. However, in most cases, designers have to sort through all possible factors and parameters of devices. The involvement of experts for questionnaire survey will reduce the time range of designing. The results of the study show a high consistency of experts' opinions with the Kendall coefficient value of 0.782 . Research materials in the future will allow planning experiments only with the most important factors. This will adjust the protocols for testing new optoelectronic systems and significantly reduce costs.
\end{abstract}

\section{Introduction}

Foresight as a tool is used in technological research recently [1]. The basis of foresight is expert methods that allow randomized and rapid assessment of the opinion of competent experts on the importance of design factors. The range of practical issues is infinitely wide, in particular, the authors attempted to "analyze hierarchies" to support decision making in microelectronics [2]. The applicability of expert assessments in the modeling of technical systems $[3,4]$ proved its feasibility and effectiveness. When designing a technical system in the process of separation of forest seeds. The grading of particles (seed material with small grain size) by spectrometric properties [5], is positioned as an alternative to traditional methods of separation (using a sieve, separation, aspiration devices). The combination of this, as well as the seeds provenance and of nursery production method [6] will further improve the seedlings quality. The variety of technical means implementing optical technology leads to the need for analytical studies of their operational parameters [4].

This technology can be used in the primary processing of reproductive material in forestry and agriculture and fully comply with modern principles of non-destructive quality control $[7,8]$. The aim of the study is to identify the applicability of one of the foresight techniques for optimizing the design of a technical system presented in the form of a optoelectronic separator.

\footnotetext{
*Corresponding author: niksal76@mail.ru
} 


\section{Materials and Methods}

As the main method of foresight, the Saaty's analytic hierarchy process [9] was used. In the analysis of hierarchies, Kendall's coefficient of concordance W [10] was used as an indicator of consensus. Its use is advisable in research on transport, in agriculture and forestry, information systems and many other areas when assessing the degree of expediency of the use of certain factors in the design of a technical system. First, the coefficient of concordance was calculated for the entire set of experts. Then one of the experts was excluded and the coefficient of concordance was calculated again for the remaining ones. Subsequently, the above procedures were carried out for all other expert assessments. In order to keep the unity of opinions, the hierarchies of that expert were excluded, with the exclusion of which the maximum increase in concordance was observed.

An average prior rank diagram of the distribution of factors reflecting the collective opinion of experts was built, and the significance of each factor was assessed. Here, the factors were plotted on the abscissa axis in descending order of their rank, and on the ordinate axis - the sum of ranks for the corresponding factor.

\section{Results}

Grading in optical features of seeds is positioned as an alternative to the traditional methods of separation (using sieve, separation, aspiration devices). The variety of technical equipment that implements optical technology to the need for analytical studies of their operational parameters. It is often necessary to analyze a fairly large number of factors and parameters, which, when studying technical systems, especially in interaction with biological objects in the form of forest reproductive material, can lead to a huge number of experiments.

Therefore, taking into account the specifics of research, it is necessary to use foresight, attracting experts from among scientists in the mechanization of forestry and agriculture, as well as develop questionnaires for the most complete and reliable analysis of factors. In the study of the technological process of the optoelectronic device [4], 11 factors were identified (Table 1). Using a table of random numbers, they were arranged randomly in questionnaires. The factors were ranked by the competent scientists in this matter.

Next, the authors processed questionnaires received from 10 experts. Table 2 illustrates the use of the rank matrix for expert analysis of the experimental sample of optoelectronic grader. With its help, the coefficient of concordance $W$, which is an estimate of the degree of consistency of opinions of researchers covered by the question, was calculated by the equation

$$
W=\frac{S}{1 / 12 m^{2}\left(n^{3}-n\right)-\sum_{j=1}^{m} T_{i}}
$$

where $S$ - the sum of squared deviations;

$m$ - the number of interviewed experts, $m=10$;

$n$ - the number of factors, $n=11$;

$$
T_{i}=\frac{1}{12} \sum_{j=1}^{m}\left(t_{j}^{3}-t_{j}\right)
$$

where $t_{j}-$ the $j$-th number of identical ranks in the $\mathrm{j}$-th ranking.

The sum of the squared deviations was calculated by the equation 


$$
S=\sum_{i=1}^{m}\left(\sum_{j=1}^{m} a_{i j}-L\right)^{2}
$$

where $a_{i j}$-rank (order number in the survey) of $i$-th factor of the $j$-th specialist;

Table 1. The main factors affecting the operating process of the optoelectronic grader.

\begin{tabular}{|c|c|}
\hline Code Number & Name \\
\hline 1 & The wavelength of the reflected radiation \\
\hline 3 & The wavelength of the absorbed radiation \\
\hline 4 & The height of the seeds pipeline \\
\hline 5 & Operating time of stepper motor \\
\hline 6 & The seeds pipeline material \\
\hline 7 & The response time of the analog-to-digital converter \\
\hline 8 & The refractive index of the seeds pipeline material \\
\hline 9 & Radius of curvature of the rotating section \\
\hline 10 & Friction coefficient of rotating section material \\
\hline 11 & Sectional shape of the pipeline \\
\hline
\end{tabular}

Table 2. Matrix of rank correlation of expert analysis of the optoelectronic grader.

\begin{tabular}{|c|c|c|c|c|c|c|c|c|c|c|c|}
\hline \multirow{2}{*}{ Experts } & \multicolumn{11}{|c|}{ Factors } \\
\hline & 1 & 2 & 3 & 4 & 5 & 6 & 7 & 8 & 9 & 10 & 11 \\
\hline 1 & 1 & 2 & 3 & 6 & 5 & 4 & 7 & 8 & 9 & 10 & 11 \\
\hline 2 & 2 & 3 & 1 & 4 & 5 & 6 & 7 & 8 & 9 & 10 & 11 \\
\hline 3 & 2 & 1 & 4 & 6 & 9 & 3 & 5 & 11 & 10 & 8 & 7 \\
\hline 4 & 3 & 1 & 2 & 8 & 6 & 4 & 7 & 9 & 5 & 11 & 10 \\
\hline 5 & 1 & 2 & 3 & 5 & 4 & 7 & 9 & 10 & 6 & 11 & 8 \\
\hline 6 & 1 & 2 & 2 & 2 & 3 & 2 & 1 & 3 & 4 & 4 & 5 \\
\hline 7 & 2 & 1 & 3 & 4 & 5 & 8 & 7 & 6 & 10 & 9 & 11 \\
\hline 8 & 1 & 2 & 3 & 4 & 6 & 5 & 7 & 8 & 11 & 9 & 10 \\
\hline 9 & 1 & 2 & 3 & 4 & 5 & 6 & 7 & 9 & 8 & 11 & 10 \\
\hline 10 & 2 & 1 & 3 & 5 & 4 & 7 & 6 & 8 & 9 & 10 & 11 \\
\hline$\sum_{j=1}^{m} a_{i j}$ & 16 & 17 & 27 & 48 & 52 & 52 & 63 & 80 & 81 & 93 & 94 \\
\hline$\Delta_{i}=\sum_{j=1}^{m} a_{i j}-L$ & -41 & -40 & -30 & -9 & -5 & -5 & 6 & 23 & 24 & 36 & 37 \\
\hline$\Delta_{i}^{2}$ & 1651 & 1571 & 878 & 75 & 21 & 21 & 40 & 546 & 594 & 1322 & 1396 \\
\hline$T_{i}=10,5$ & $=56$, & & $=8$ & 5,55 & & & & $n^{3}-$ & $=1$ & $20 ; W$ & 0,782 \\
\hline
\end{tabular}

$L$ - average value of the sum of ranks for each factor

$$
L=\frac{\sum_{j=1}^{n} \sum_{j=1}^{m} a_{i j}}{n}
$$


After calculating the Kendall's coefficient of concordance W, its significance was determined by criterion $\chi^{2}$, comparing its calculated and tabular values, since the value $m(n-1) W$ has $\chi^{2}$-distribution with the number of degrees of freedom $f=n-1$.

The calculated value of the $\chi^{2}$-distribution was determined by the equation

$$
\chi^{2}=\frac{S}{1 / 12 m n(n+1)-\frac{1}{n-1} \sum_{j=1}^{m} T_{i}}
$$

In our case $\chi_{\text {calc }}^{2}=74,5$. From statistical tables, we find that for a $5 \%$ level of significance with $f=11-1=10$ degrees of freedom, the value of $\chi_{t a b}^{2}=18.307$. Since the value of $\chi^{2}$-distribution calculated by the formula (3) is larger than tabular one with an appropriate number of degrees of freedom, we are $95 \%$ likely to say that expert opinion on the degree of influence of factors is assessed by the coefficient of concordance $\mathrm{W}=0.782$ and the consistency of scientists is not random.

Fig. 1 shows the average prior diagram of the ranks of the distribution of factors, reflecting the collective opinion of experts on the hierarchy of factors affecting the performance of the optoelectronic grader.

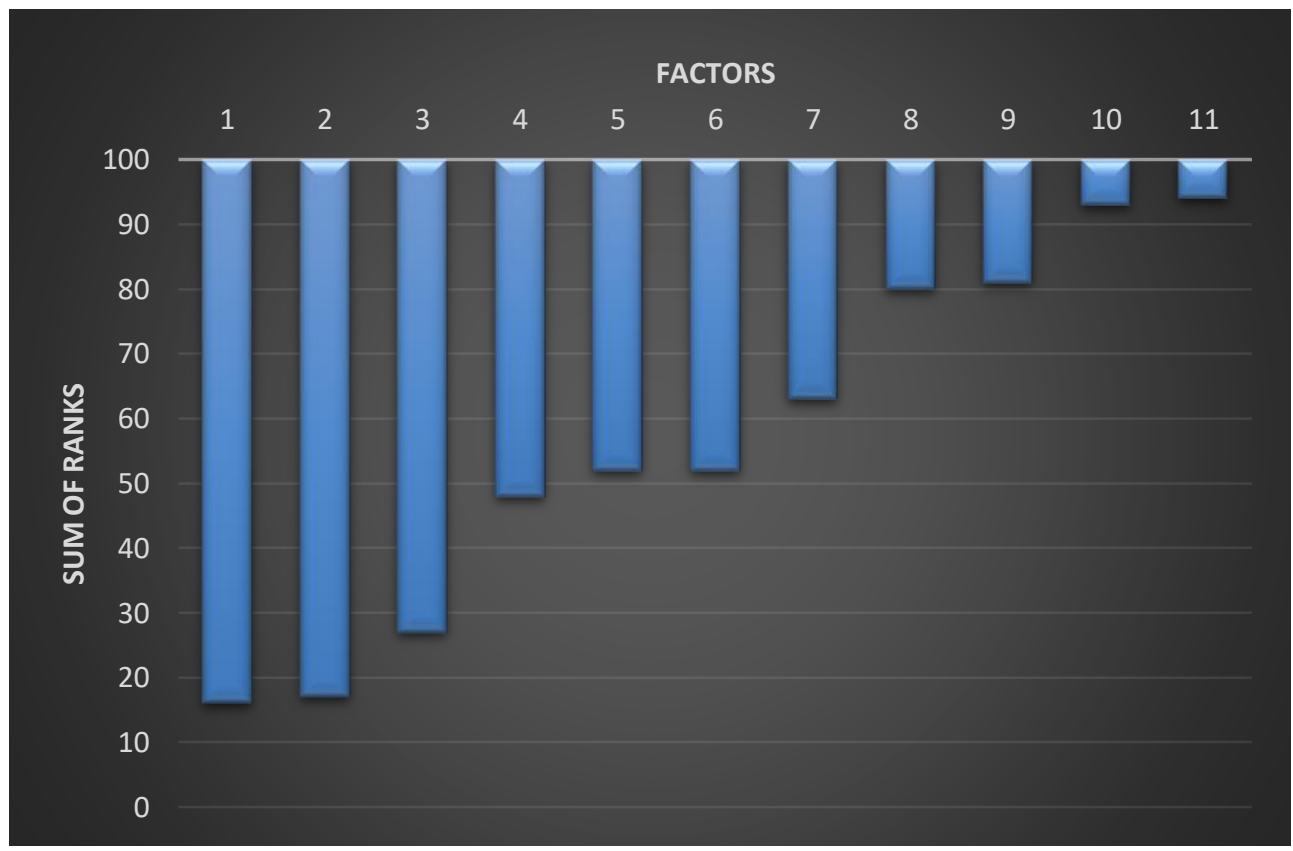

Fig. 1. Average prior rank diagram.

Based on prior ranking, taking into account the results of the conducted psychological experiment, 1, 2 and 3 factors (see Table 2) were selected for further simulation - the wavelength of the reflected radiation, the wavelength of the absorbed radiation, the height of the seeds pipeline. With a certain degree of probability, it can be assumed that they most strongly affect the quality indicators of the technological process of an optoelectronic device. 


\section{Discussions}

Definitely, foresight with the study of the opinions of scientists in the form of brainstorming and expert analysis helps in making decisions about the feasibility of choosing direction in the design of technical systems. However, it is not always possible to find a sufficiently large number of experts in this area due to its limited distribution. In this case, it is necessary to answer the question about the optimal number of experts and refer them only to a narrow circle of specialists of the required area, or the possible questionnaire among specialists with technical education. To this end, it is necessary to plan research with the involvement of a wide range of experts. However, even with a small number of experts, using the analytic hierarchy process yields a rather high coefficient of concordance -0.782 (the maximum value is 1.0 ).

\section{Conclusion}

The use of the hierarchy analysis method in the planning of the optoelectronic grader experiment a priori allowed:

- reduce the time interval when planning it;

- select the most important factors from the perspective of competent experts;

- compensate for part of the cost of searching all possible combinations of factors

- lay the groundwork for future planning of the multifactor experiment.

\section{References}

1. N. Li, K. Chen, M. Kou, Technol. Forecast. Soc. Change 119, 246-255 (2017)

2. T. Novikova, A. Shevchenko, Proceedings of the 33rd International Business Information Management Association Conference, IBIMA 2019: Education Excellence and Innovation Management through Vision 2020, 2914-2920 (2019)

3. A. Spickermann, V. Grienitz, H. von der Gracht, Technol. Forecast. Soc. Change 89, 201-221 (2014)

4. A. Novikov, M. Drapalyuk, O. Dornyak, V. Zelikov, V. Ivetić, Inventions 4, 55 (2019)

5. M. Jabłoński, P. Tylek, J. Walczyk, R. Tadeusiewicz, A. Piłat, Sensors 16, 1319 (2016)

6. V. Ivetić, M. Škorić, Ann. For. Res. 56, 297-306 (2013).

7. M. Tigabu, P. Odén, Seed Sci. Technol. 32, 593-606 (2004)

8. S. Li, H. Luo, M. Hu, M. Zhang, J. Feng, Y. Liu, Q. Dong, B. Liu, Artif. Intell. Agric. 2, 85-98 (2019)

9. T. Saaty, Fuzzy Sets Syst. 1, 57-68 (1978)

10. J. Teles, J. Appl. Stat. 39, 1749-1764 (2012) 